\title{
Monotonic Properties of Generalized Nielsen's $\beta$-function
}

\author{
Jumei Zhang, Li Yin*, Wenyan Cui \\ School of Science, Binzhou University \\ *Corresponding author: yinli7979@163.com
}

Received December 10, 2018; Revised February 04, 2019; Accepted February 23, 2019

Abstract In the paper, we discuss a new $k$-generalization of the Nielsen's $\beta$-function. Later, we study the completely monotonicity, convexity and inequalities of the new function.

Keywords: Nielsen's $\beta$-function, $k$-generalization, inequality, completely monotonic

2010 Mathematics Subject Classification. Primary: 39B12. Secondary: 34A25, 30D05.

Cite This Article: Jumei Zhang, Li Yin, and Wenyan Cui, “Monotonic Properties of Generalized Nielsen's $\beta$-function.” Turkish Journal of Analysis and Number Theory, vol. 7, no. 1 (2019): 18-22. doi: 10.12691/tjant-7-1-4.

\section{Introduction}

The Nielsen's $\beta$-function is defined as ([1-5])

$$
\begin{aligned}
\beta(x) & =\int_{0}^{1} \frac{t^{x-1} d t}{1+t} d t \\
& =\int_{0}^{\infty} \frac{e^{-x t}}{1+e^{-t}} d t \\
& =\sum_{n=0}^{\infty} \frac{(-1)^{n}}{n+x} \\
& =\frac{1}{2}\left\{\psi\left(\frac{x+1}{2}\right)-\psi\left(\frac{x}{2}\right)\right\}
\end{aligned}
$$

where $x \in(0, \infty), \quad \psi(x)=\frac{d}{d x} \ln \Gamma(x)$ is the digamma or psi function and $\Gamma(x)$ is the Euler's gamma function. It is well known that

$$
\begin{gathered}
\beta(x+1)=\frac{1}{x}-\beta(x), \\
\beta(x)+\beta(1-x)=\frac{\pi}{\sin \pi x} .
\end{gathered}
$$

The Nielsen's $\beta$-function has been deeply researched in past years. Such as, K. Nantomah studied the properties and inequalities of the function in [3], gave some convexities and monotonicity of the function in [6], and obtained some convexity, monotonicity and inequalities involving a generalized form of the Wallis's cosine formula in [7]. The function can be used to calculate some integrals [4,8]. Recently, K. Nantomah studied the properties and inequalities of a $p$-generalization of the
Nielsen's function in [5]. In this paper, we investigate a $k$-generalization of the Nielsen's $\beta$-function function. The notations $\mathbf{N}=\{1,2,3,4, \cdots\}$ and $\mathbf{N}_{0}=\mathbf{N} \cup\{0\}$.

The $k$-analogue of the gamma function is defined as

$$
\Gamma_{k}(x)=\int_{0}^{\infty} t^{x-1} e^{-\frac{t^{k}}{k}} d t,
$$

where $k \in \mathbf{N}, \quad x \in(0, \infty)$ and $\Gamma_{1}(x)=\Gamma(x)$.

Remark 1.1. For $x>0$ and $k \in \mathbf{N}$, the k-analogue of the Gamma function satisfies
1. $\Gamma_{k}(k)=1$,
2. $\Gamma_{k}(x+k)=x \Gamma_{k}(x)$.

\section{The New Generalized Nielsen's $\beta$-function}

In this part, we define a $k$-generalization of the Nielsen's $\beta$-function and study some of its properties.

Definition 2.1. We define the k-generalization of the Nielsen's $\beta$-function as

$$
\begin{aligned}
\beta_{k}(x) & =\int_{0}^{1} \frac{t^{x-1}}{1+t^{k}} d t \\
& =\int_{0}^{\infty} \frac{e^{-x t}}{1+e^{-k t}} d t \\
& =\sum_{n=0}^{\infty}\left(\frac{1}{2 n k+x}-\frac{1}{2 n k+k+x}\right) \\
& =\frac{1}{2}\left\{\psi_{k}\left(\frac{x+k}{2}\right)-\psi_{k}\left(\frac{x}{2}\right)\right\}
\end{aligned}
$$


where $x \in(0, \infty), \psi_{k}(x)=\frac{d}{d x} \ln \Gamma_{k}(x)$ is the digamma or psi function and $\Gamma_{k}(x)$ is defined in (7).

Some special values of the $k$-generalization function $\beta_{k}(x)$ can be calculated, for example

$$
\begin{aligned}
& \beta_{k}(k)=\frac{\ln 2}{k}, \beta_{k}\left(\frac{k}{2}\right)=\frac{\pi}{2 k}, \\
& \beta_{k}\left(\frac{3 k}{2}\right)=\frac{2}{k}-\frac{\pi}{2 k}, \beta_{k}(2 k)=\frac{1}{k}-\frac{\ln 2}{k} .
\end{aligned}
$$

Theorem 2.2. The k-generalization function $\beta_{k}(x)$ satisfies the equality for $x>0$ and $k \in \mathbf{N}$

$$
\beta_{k}(x+k)+\beta_{k}(x)=\frac{1}{x} .
$$

Proof.

$$
\begin{aligned}
& \beta_{k}(x+k)+\beta_{k}(x) \\
= & \int_{0}^{\infty} \frac{e^{-(x+k) t}}{1+e^{-k t}} d t+\int_{0}^{\infty} \frac{e^{-x t}}{1+e^{-k t}} d t \\
= & \int_{0}^{\infty} e^{-x t} d t \\
= & -\frac{1}{x} \cdot\left[e^{-x t}\right]_{0}^{\infty}=\frac{1}{x} .
\end{aligned}
$$

The proof is finished.

By (12), we achieve

$$
0<\beta_{k}(x) \leq \frac{1}{x},
$$

and the relationship

$$
\begin{aligned}
& \beta_{k}(x+m \cdot k) \\
= & \sum_{l=0}^{m-1} \frac{(-1)^{l+m+1}}{x+l}+(-1)^{m} \beta_{k}(x),(m \in \mathbf{N}) .
\end{aligned}
$$

Theorem 2.3. For $x>0$ and $k \in \mathbf{N}$,

(1) $\beta_{k}^{(m)}(x)$ is positive and decreasing if $m \in \mathbf{N}_{0}$ is even;

(2) $\beta_{k}^{(m)}(x)$ is negative and increasing if $m \in \mathbf{N}_{0}$ is odd;

(3) $\left|\beta_{k}^{(m)}(x)\right|$ is decreasing for any $m \in \mathbf{N}_{0}$ and

$$
\left|\beta_{k}^{(m)}(x)\right| \leq \frac{m !}{x^{m+1}} .
$$

Proof. Using (9), we get the recursive formula

$$
\beta_{k}^{(m)}(x)=\int_{0}^{\infty} \frac{(-1)^{m} t^{m} e^{-x t}}{1+e^{-k t}} d t .
$$

Then we can achieve the conclusion.

Corollary 2.4. The k-generalization function $\beta_{k}(x)$ has the following properties for $x>0$ and $k \in \mathbf{N}$

(1) $\beta_{k}(x)$ is completely monotonic;

(2) $\beta_{k}^{(m)}(x)$ is completely monotonic if $m \in \mathbf{N}_{0}$ is even;
(3) $-\beta_{k}^{(m)}(x)$ is completely monotonic if $m \in \mathbf{N}_{0}$ is odd.

Proof. Using (15), we get

$$
(-1)^{m} \beta_{k}^{(m)}(x)=\int_{0}^{\infty} \frac{t^{m} e^{-x t}}{1+e^{-k t}} d t \geq 0 .
$$

Then we achieve the conclusion.

Theorem 2.5. The k-generalization function $\beta_{k}(x)$ is logarithmical convex for $x>0$ and $k \in \mathbf{N}$.

Proof. Let $a>1, b>1$, and $\frac{1}{a}+\frac{1}{b}=1$, for $x>0$ and $y>0$, by the Hölder's inequality, we can get

$$
\begin{aligned}
& \beta_{k}\left(\frac{x}{a}+\frac{y}{b}\right) \\
= & \int_{0}^{\infty} \frac{e^{-\left(\frac{x}{a}+\frac{y}{b}\right) t}}{1+e^{-k t}} d t \\
= & \int_{0}^{\infty}\left(\frac{e^{-x t}}{1+e^{-k t}}\right)^{\frac{1}{a}}\left(\frac{e^{-y t}}{1+e^{-k t}}\right)^{\frac{1}{b}} d t \\
\leq & \left(\int_{0}^{\infty} \frac{e^{-x t}}{1+e^{-k t}} d t\right)^{\frac{1}{a}}\left(\int_{0}^{\infty} \frac{e^{-y t}}{1+e^{-k t}} d t\right)^{\frac{1}{b}} \\
= & \left(\beta_{k}(x)\right)^{\frac{1}{a}}\left(\beta_{k}(y)\right) \frac{1}{b} .
\end{aligned}
$$

From the definition of logarithmical convex, we can achieve the conclusion.

Based on the above, we could infer the followings:

Corollary 2.6. The k-generalization function $\beta_{k}(x)$ satisfies

$$
\left|\beta_{k}^{\left(\frac{m}{a}+\frac{n}{b}\right)}\left(\frac{x}{a}+\frac{y}{b}\right)\right| \leq\left|\beta_{k}^{(m)}(x)\right| \frac{1}{a}\left|\beta_{k}^{(n)}(y)\right|^{\frac{1}{b}},
$$

for $a>1, \quad b>1, \quad x>0, \quad y>0, \quad k \in \mathbf{N}, \quad m, n \in \mathbf{N}_{0}$ and $\frac{1}{a}+\frac{1}{b}=1$.

Proof. Using (15) and the similar procedure in Theorem 2.5, we can get the inequality (17).

Remark 2.7. When $m=n$ is even in Corollary 2.6, then $\beta_{k}(x)$ satisfies

$$
\beta_{k}^{(m)}\left(\frac{x}{a}+\frac{y}{b}\right) \leq\left(\beta_{k}^{(m)}(x)\right)^{\frac{1}{a}}\left(\beta_{k}^{(m)}(y)\right)^{\frac{1}{b}} .
$$

Especially, let $a=b=2, x=y$ and $n=m+2$ in Corollary 2.6, we achieve the Turan-Type inequality

$$
\left|\beta_{k}^{(m+1)}(x)\right|^{2} \leq\left|\beta_{k}^{(m+2)}(x)\right|\left|\beta_{k}^{(m)}(x)\right|
$$

Moreover, if $m=0$ in (18), we have the famous inequality

$$
\left(\beta_{k}^{\prime}(x)\right)^{2} \leq \beta_{k}^{\prime \prime}(x) \beta_{k}(x)
$$


which implies the function $\frac{\beta_{k}^{\prime}(x)}{\beta_{k}(x)}$ is increasing for $x>0$.

Corollary 2.8. The k-generalization function $\beta_{k}(x)$ satisfies

$$
\begin{gathered}
{\left[\beta_{k}(x+y)\right]^{2} \leq \beta_{k}(x) \beta_{k}(y),} \\
\beta_{k}(x+y) \leq \beta_{k}(x)+\beta_{k}(y),
\end{gathered}
$$

for $x>0, y>0$.

Proof. Since $\beta_{k}(x)$ is decreasing, we get

$$
\beta_{k}(x+y)<\beta_{k}\left(\frac{x+y}{2}\right) .
$$

Let $a=b=2$ in Theorem 2.5, we achieve

$$
\beta_{k}\left(\frac{x+y}{2}\right) \leq \sqrt{\beta_{k}(x) \beta_{k}(y)}
$$

then we can get

$$
\beta_{k}(x+y)<\sqrt{\beta_{k}(x) \beta_{k}(y)} .
$$

By the basic AM-GM inequality, we get

$$
\begin{aligned}
& \beta_{k}(x+y)<\sqrt{\beta_{k}(x) \beta_{k}(y)} \\
\leq & \frac{\beta_{k}(x)}{2}+\frac{\beta_{k}(y)}{2} \leq \beta_{k}(x)+\beta_{k}(y) .
\end{aligned}
$$

These imply that the inequalities (20) and (21) are right. Corollary 2.9. The formula

$$
1<\frac{\beta_{k}(u)}{\beta_{k}(u+1)}<\frac{\beta_{k}(u-1)}{\beta_{k}(u)}
$$

satisfies for $u>1$ and $k \in \mathbf{N}$.

Proof. Since $\beta_{k}(x)$ is decreasing and positive, so $\beta_{k}(u+1)<\beta_{k}(u)$, which implies

$$
1<\frac{\beta_{k}(u)}{\beta_{k}(u+1)} .
$$

Let $x=u-1$ and $y=u+1$ in (20), we achieve

then we can get

$$
\beta_{k}^{2}(u)<\beta_{k}(u-1) \beta_{k}(u+1) \text {, }
$$

$$
\frac{\beta_{k}(u)}{\beta_{k}(u+1)}<\frac{\beta_{k}(u-1)}{\beta_{k}(u)} .
$$

These imply that the inequality (22) is right.

Theorem 2.10. For $x>0$ and $k \in \mathbf{N}, \quad \beta_{k}(x)$ satisfies the inequality

$$
\begin{aligned}
& \exp \left\{\beta_{k}\left(x+\frac{k}{2}\right)\right\} \\
& \leq\left(\frac{\Gamma_{k}\left(\frac{x}{2}+k\right) \Gamma_{k}\left(\frac{x}{2}\right)}{\Gamma_{k}^{2}\left(\frac{x+k}{2}\right)}\right)^{\frac{1}{k}} \leq \exp \left\{\frac{1}{2 x}\right\} .
\end{aligned}
$$

Proof. Theorem 2.5 implies that $\beta_{k}(x)$ is convex. So $\beta_{k}(x)$ satisfies the inequality

$$
\beta_{k}\left(\frac{a+b}{2}\right) \leq \frac{1}{b-a} \int_{a}^{b} \beta_{k}(s) d s \leq \frac{\beta_{k}(a)+\beta_{k}(b)}{2} .
$$

In view of (11) and setting $a=x>0, b=x+k$, becomes

$$
\begin{aligned}
& \beta_{k}\left(x+\frac{k}{2}\right) \\
\leq & \frac{1}{k}\left[\ln \Gamma_{k}\left(\frac{u+k}{2}\right)-\ln \Gamma_{k}\left(\frac{u}{2}\right)\right]_{X}^{x+k} \\
\leq & \frac{\beta_{k}(x+k)+\beta_{k}(x)}{2} .
\end{aligned}
$$

Taking the exponent of the inequality, we achieve the desired result.

Theorem 2.11. For $x>0$ and $k \in \mathbf{N}$, the function $g(x)=x \beta_{k}(x)$ is completely monotonic, decreasing and convex.

Proof. By calculating, we can get

$$
g^{(m)}(x)=m \beta_{k}^{(m-1)}(x)+x \beta_{k}^{(m)}(x) .
$$

By the convolution theorem for Laplace transforms, we achieve

$$
\begin{aligned}
& \frac{(-1)^{m} g^{(m)}(x)}{x} \\
= & (-1)^{m}\left[\frac{m}{x} \beta_{k}^{(m-1)}(x)+\beta_{k}^{(m)}(x)\right] \\
= & -m \int_{0}^{\infty} e^{-x t} d t \cdot \int_{0}^{\infty} \frac{t^{m-1} e^{-x t}}{1+e^{-k t}} d t+\int_{0}^{\infty} \frac{t^{m} e^{-x t}}{1+e^{-k t}} d t \\
= & -m \int_{0}^{\infty}\left[\int_{0}^{t} \frac{s^{m-1}}{1+e^{-k s}} d s\right] e^{-x t} d t+\int_{0}^{\infty} \frac{t^{m} e^{-x t}}{1+e^{-k t}} d t \\
= & \int_{0}^{\infty} G(t) e^{-x t} d t,
\end{aligned}
$$

where

$$
\begin{gathered}
G(t)=-m \int_{0}^{t} \frac{s^{m-1}}{1+e^{-k s}} d s+\frac{t^{m}}{1+e^{-k t}}, \\
G^{\prime}(t)=-m \frac{t^{m-1}}{1+e^{-k t}}+m \frac{t^{m-1}}{1+e^{-k t}}+k \frac{t^{m} e^{-k t}}{\left(1+e^{-k t}\right)^{2}}>0,
\end{gathered}
$$

and $G(0)=\lim _{t \rightarrow 0} G(t)=0$. Then, for $t>0$, we achieve $G(t)>G(0)=0$

So

$$
(-1)^{m} g^{(m)}(x)>0,
$$

which implies that $g(x)$ is completely monotonic.

Letting $m=1$ in (29), we achieve

$$
(-1) g^{\prime}(x)=-\beta_{k}(x)-x \beta_{k}^{\prime}(x)>0 .
$$

That is 


$$
g^{\prime}(x)=\beta_{k}(x)+x \beta_{k}^{\prime}(x)<0,
$$

which implies $g(x)$ is decreasing, and let $m=2$ in (29), we can get

$$
g^{\prime \prime}(x)=2 \beta_{k}^{\prime}(x)+x \beta_{k}^{\prime \prime}(x)>0,
$$

which implies that $g(x)$ is convex.

Corollary 2.12. The function $h(x)=x \beta_{k}^{\prime}(x)$ is increasing and convex for $x>0$.

Proof. Based on (29), (31) and Theorem 2.3, we can achieve

$$
h^{\prime}(x)=\beta_{k}^{\prime}(x)+x \beta_{k}^{\prime \prime}(x)>2 \beta_{k}^{\prime}(x)+x \beta_{k}^{\prime \prime}(x)>0,
$$

and

$$
h^{\prime \prime}(x)=2 \beta_{k}^{\prime \prime}(x)+x \beta_{k}^{\prime \prime \prime}(x)<3 \beta_{k}^{\prime \prime}(x)+x \beta_{k}^{\prime \prime \prime}(x)<0,(33)
$$

which implies that $h(x)=x \beta_{k}^{\prime}(x)$ is increasing and convex for $x>0$.

Corollary 2.13. For $x>0, y>1$ and $k \in \mathbf{N}$, the inequality

$$
\beta_{k}(x y) \leq \beta_{k}(x)+\beta_{k}(y)
$$

is right.

Proof. For $x>0, y>1$, set

$$
f(x, y)=\beta_{k}(x y)-\beta_{k}(x)-\beta_{k}(y),
$$

then

$$
\frac{\partial f(x, y)}{\partial x}=y \beta_{k}^{\prime}(x y)-\beta_{k}^{\prime}(x)=\frac{1}{x}\left[x y \beta_{k}^{\prime}(x y)-x \beta_{k}^{\prime}(x)\right] .
$$

Since $x y>x$ and $x \beta_{k}^{\prime}(x)$ is increasing, we can get $\frac{\partial f(x, y)}{\partial x} \geq 0$, which implies $f(x, y)$ is increasing. So, for $\forall x \in(0, \infty)$, we can achieve

$$
f(x, y) \leq \lim _{x \rightarrow \infty} f(x, y)=-\beta_{k}(y)<0,
$$

hence $\beta_{k}(x y) \leq \beta_{k}(x)+\beta_{k}(y)$.

Theorem 2.14. For $x>0, \quad m \in \mathbf{N}_{0}$ and $k \in \mathbf{N}$, the function

$$
g_{m}(x)=\frac{x^{m+1}}{m !}\left|\beta_{k}^{(m)}(x)\right| \text {. }
$$

Hence,

(1) $\lim _{x \rightarrow 0} g_{m}(x)=1$ and $\lim _{x \rightarrow 0} g_{m}^{\prime}(x)=0$,

(2) $g_{m}(x)$ is decreasing.

Proof. By Theorem 2.3, we could infer

$$
\left|\beta_{k}^{(m)}(x+k)\right| \leq \frac{m !}{(x+k)^{m+1}},
$$

and

$$
\beta_{k}^{(m)}(x+k)+\beta_{k}^{(m)}(x)=\frac{m !}{x^{m+1}},
$$

so, for $x>0, \quad m \in \mathbf{N}_{0}$ and $k \in \mathbf{N}$,

$$
\lim _{x \rightarrow 0} \frac{x^{m+1}}{m !}\left|\beta_{k}^{(m)}(x+k)\right| \leq \lim _{x \rightarrow 0} \frac{x^{m+1}}{m !} \frac{m !}{(x+k)^{m+1}}=0,
$$

where $0 !=1$. Then

$$
\begin{aligned}
& \lim _{x \rightarrow 0} g_{m}(x)=\lim _{x \rightarrow 0} \frac{x^{m+1}}{m !}\left|\beta_{k}^{(m)}(x)\right| \\
= & \lim _{x \rightarrow 0}\left\{1-\frac{x^{m+1}}{m !}\left|\beta_{k}^{(m)}(x+k)\right|\right\}=1,
\end{aligned}
$$

and

$$
\begin{aligned}
& \lim _{x \rightarrow 0} g_{m}^{\prime}(x) \\
= & \lim _{x \rightarrow 0}\left\{\frac{(m+1) x^{m}}{m !}\left|\beta_{k}^{(m)}(x)\right|-\frac{x^{m+1}}{m !}\left|\beta_{k}^{(m+1)}(x)\right|\right\} \\
= & \lim _{x \rightarrow 0} h(x)=0,
\end{aligned}
$$

where

$$
\begin{aligned}
h(x)= & \frac{(m+1) x^{m}}{m !}\left[\frac{m !}{x^{m+1}}-\left|\beta_{k}^{(m)}(x+k)\right|\right] \\
& -\frac{x^{m+1}}{m !}\left[\frac{(m+1) !}{x^{m+2}}-\left|\beta_{k}^{(m+1)}(x+k)\right|\right] .
\end{aligned}
$$

By the convolution theorem for Laplace transforms, we achieve

$$
\begin{aligned}
& \frac{m !}{x^{m+1}} g_{m}^{\prime}(x) x \\
= & \frac{m+1}{x}\left|\beta_{k}^{(m)}(x)\right|-\left|\beta_{k}^{(m+1)}(x)\right| \\
= & (m+1) \int_{0}^{\infty} e^{-x t} d t \int_{0}^{\infty} \frac{t^{m} e^{-x t}}{1+e^{-k t}} d t-\int_{0}^{\infty} \frac{t^{m+1} e^{-x t}}{1+e^{-k t}} d t \\
= & (m+1) \int_{0}^{\infty}\left[\int_{0}^{t} \frac{s^{m}}{1+e^{-k s}} d s\right] e^{-x t} d t-\int_{0}^{\infty} \frac{t^{m+1} e^{-x t}}{1+e^{-k t}} d t \\
= & \int_{0}^{\infty} G_{m}(t) e^{-x t} d t,
\end{aligned}
$$

where

$$
G_{m}(t)=(m+1) \int_{0}^{t} \frac{s^{m}}{1+e^{-k s}} d s-\frac{t^{m+1}}{1+e^{-k t}},
$$

$$
\begin{aligned}
G_{m}^{\prime}(t) & (m+1) \frac{t^{m}}{1+e^{-k t}}-(m+1) \frac{t^{m}}{1+e^{-k t}}-k \frac{t^{m} e^{-k t}}{\left(1+e^{-k t}\right)^{2}}
\end{aligned}
$$

$<0$,

and $G_{m}(0)=\lim _{t \rightarrow 0} G_{m}(t)=0$. Then, for $t>0$, we achieve $G_{m}(t)<G_{m}(0)=0$.

$$
g_{m}^{\prime}(x)<0
$$

which implies that $g_{m}(x)$ is decreasing. 


\section{Conclusion}

In the paper, we defined a new $k$-generalization function of the Nielsen's $\beta$-function, proved the new $k$-generalization function is convex, decreasing and completely monotonic as well as conducted some inequalities related with the function. The results can be used to evaluate or estimate some integrals. Moreover, the conclusions would play important role in the further study of the function.

\section{Thanks}

The authors are grateful to anonymous referees and the editor for their careful corrections and valuable comments on the original version of this paper.

\section{Funding}

This work was supported by National Natural Science Foundation of China (Grant No.11601036, Grant No.11701320 and 11705122), the Science and Technology Foundations of Shandong Province (Grant No.
J16li52, J14li54 and J17KA161) and Science Foundations of Binzhou University (Grant No. BZXYFB20150903 and BZXYL1704).

\section{References}

[1] D. F. Connon, On an integral involving the digamma function. arXiv: 1212. 1432 [math.GM]

[2] I. S. Gradshteyn and I. M. Ryzhik, Table of Integrals, Series and Products. Edited by D. Zwillinger and V. Moll. Academic Press, New York, Sth Edition, 2014.

[3] K. Nantomah, On Some Properties and Inequalities for the Nielsen's $\beta$ Function, arXiv: 1708. 06604vl 1432 [math.CA], 12 Pages.

[4] N. Nielsen, Handbuch der Theorie der Gammafunction, First Edition, Leipzig: B. G. Teubner, 1906.

[5] K. Nantomah, A generalization of the Nielsen's $\beta$-function. Probl. Anal. Issue 1998-6262 (2)(2018), 16-26.

[6] K. Nantomah, Monotonicity and convexity properties of the Nielsen's $\beta$-function. Probl. Anal. Issues Anal. 6(24)(2) (2017), 81-93.

[7] K. Nantomah, Monotonicity and convexity properties and some inequalities involving a generalized form of the Wallis' cosine formula. Asian Research Journal of Mathematics, 6(3)(2017), 1-10

[8] Boyadzhiev K. N., Medina L. A., and Moll V. H. The integrals in Gradshteyn and Ryzhik, Part II: The incomplete beta function. Scientia, Ser. A, Math. Sci., 2009, vol. 18. pp. 61-75.

C The Author(s) 2019. This article is an open access article distributed under the terms and conditions of the Creative Commons Attribution (CC BY) license (http://creativecommons.org/licenses/by/4.0/). 